\title{
Epithelial cell adhesion molecule is overexpressed in hypopharyngeal carcinoma and suppresses the metastasis and proliferation of the disease when downregulated
}

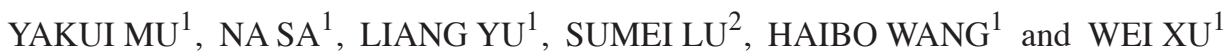 \\ ${ }^{1}$ Department of Otolaryngology-Head and Neck Surgery, Provincial Hospital Affiliated to Shandong University, Jinan, \\ Shandong 250021; ${ }^{2}$ Department of Laboratory Medicine, Shandong Provincial Qianfoshan Hospital, \\ Jinan, Shandong 250014, P.R. China
}

Received October 22, 2013; Accepted April 10, 2014

DOI: $10.3892 / 01.2014 .2140$

\begin{abstract}
The epithelial cell adhesion molecule (EpCAM) is overexpressed in the majority of human epithelial carcinomas, and its overexpression is associated with proliferation and neoplastic transformation. However, the precise molecular mechanism involved in EpCAM-related proliferation and metastasis in hypopharyngeal carcinoma is unknown. The aim of the present study was to identify the role of EpCAM in the metastasis and proliferation of hypopharyngeal carcinoma. An immunohistochemical staining assay indicated that EpCAM was overexpressed in primary hypopharyngeal carcinoma tissues, and that this overexpression correlated with the tumor size and lymph node metastasis. In the following treatment of the hypopharyngeal carcinoma FaDu cell line with EpCAM, the downregulation of EpCAM was found to significantly suppress cell metastasis and proliferation, as detected by Transwell, clone formation and MTT assays. Additionally, western blot analysis revealed that EpCAM downregulation increased the expression of the adhesion- and proliferation-related factors, E-cadherin, $\alpha$-catenin and $\beta$-catenin, in the cytoskeleton, as well as $\beta$-catenin expression in the nucleus. In conclusion, the present study indicated that EpCAM is a potential oncogene and contributes to the metastasis of hypopharyngeal carcinoma. The current study is the first to provide evidence for the potential value of targeting EpCAM in hypopharyngeal carcinoma therapy.
\end{abstract}

\section{Introduction}

Hypopharyngeal carcinoma, one of the most common types of head and neck squamous cell carcinoma, results in substantial

Correspondence to: Professor Wei Xu, Department of Otolaryngology-Head and Neck Surgery, Provincial Hospital Affiliated to Shandong University, 324 Jingwuweiqi Road, Jinan, Shandong 250021, P.R. China

E-mail: xuwhns@126.com

Key words: hypopharyngeal carcinoma, metastasis, proliferation, epithelial cell adhesion molecule morbidities and mortalities annually. The majority of patients with hypopharyngeal carcinoma are frequently observed to be at an advanced stage, with lymph node metastasis at the initial diagnosis. The predominant therapy for hypopharyngeal carcinoma remains confined to surgery with additional treatments of radiotherapy and chemotherapy (1). Although surgical techniques and anticancer agents have advanced, the overall survival rates have not significantly improved during the last two decades $(2,3)$. Therefore, identification of the associated target factors and the potential mechanism of hypopharyngeal carcinoma metastasis and proliferation are important for the survival of patients.

The epithelial cell adhesion molecule (EpCAM), also known as CD326, a 39-42-kDa type I trans-membrane glycoprotein, consists of an extracellular domain, a single transmembrane domain and a short 26-amino acid intracellular domain (EpICD) (4). EpCAM expression is observed at the basolateral membrane of the majority of epithelium, with the exception of squamous epithelium $(5,6)$. Furthermore, EpCAM is overexpressed in the majority of human epithelial carcinomas, including breast, colorectal, prostate, hepatic and head and neck carcinomas, and its overexpression in these cancers is associated with proliferation and neoplastic transformation (7-11). Although EpCAM is regarded as a $\mathrm{Ca}^{2+}$-independent homophilic cell-cell adhesion molecule, its intercellular adhesive activity is extremely weak $(12,13)$. EpCAM predominantly contributes to proliferation and metastasis by regulating E-cadherin mediated-adhesion and $\beta$-catenin signaling $(14,15)$. EpCAM has previously been regarded as an additional marker for the identification of cancer-initiating stem cells (16). Cancer stem cells exhibiting a high level of EpCAM expression are more tumorigenic and malignant than those exhibiting low expression levels (17). Therefore, based on the radiation and drug resistance of cancer stem cells, targeting EpCAM may present a promising approach for cancer therapy.

However, few studies have analyzed the effect of EpCAM in hypopharyngeal carcinomas and thus, knowledge regarding the role of EpCAM in the process of carcinogenesis, tumor progression and metastasis requires further elucidation. In the present study, the expression of EpCAM in hypopharyngeal 
carcinoma was examined. Furthermore, the EpCAM small interfering RNA (siRNA) was employed to downregulate EpCAM in hypopharyngeal carcinoma FaDu cells for studying the role of EpCAM and its mechanism. The present study also attempted to clarify whether EpCAM may be regarded as a potential candidate for hypopharyngeal carcinoma therapy.

\section{Materials and methods}

Tissue sections and immunohistochemistry. A total of 40 hypopharyngeal carcinoma tissue samples were obtained from patients with hypopharyngeal carcinoma at the Shandong University Affiliated Provincial Hospital (Jinan, China). For controls, samples of normal squamous epithelium tissue were obtained from the non-cancerous regions of these patients. No patients had previously received pre-operative chemotherapy or radiotherapy. Patient information, including age, gender and tumor-node-metastasis stage, were obtained from the surgical and pathological records, and written informed consent was obtained from all patients. The study was approved by the ethics committee of Shandong University (Jinan, China).

The paraffin-embedded tissue sections were hydrated in xylene (Guangcheng Chemical Reagent Co., Ltd., Tianjin, China) and a graded alcohol series. Antigen retrieval was performed in a water bath at $95^{\circ} \mathrm{C}$ for $20 \mathrm{~min}$ with citric acid buffer (Beijing Zhongshan Golden Bridge Biotechnology Co., Ltd., Beijing, China), and endogenous peroxidase activity was blocked with $3 \% \mathrm{H}_{2} \mathrm{O}_{2}$. Next, the tissue sections were incubated with goat serum (Beijing Zhongshan Golden Bridge Biotechnology Co., Ltd.) for $45 \mathrm{~min}$ and stained with rabbit anti-human EpCAM antibody (Abcam, Cambridge, MA, USA) at $4^{\circ} \mathrm{C}$ overnight. For the negative control, an equal amount of phosphate-buffered saline (PBS) was used instead of the primary antibody. Subsequent to washing the tissue section with PBS three times, the biotin-labeled goat anti-rabbit IgG primary antibody (IgG/Bio, Beijing Zhongshan Golden Bridge Biotechnology Co., Ltd.) was detected following incubation with a secondary antibody (horseradish peroxidase-labeled streptavidin; S-A/HRP, Beijing Zhongshan Golden Bridge Biotechnology Co., Ltd.) for $30 \mathrm{~min}$ at $37^{\circ} \mathrm{C}$. 3,3'-Diamino-benzidine tetrahydrochloride solution (Beijing Zhongshan Golden Bridge Biotechnology Co., Ltd.) was used to visualize positive staining, and hematoxylin was used to counterstain the nucleoli.

Cell line and cell culture. The human hypopharyngeal carcinoma FaDu cell line was obtained from the American Type Culture Collection (Manassas, VA, USA). The FaDu cells were cultured in Dulbecco's modified Eagle's medium (DMEM; Gibco-BRL, Carlsbad, CA, USA) containing 10\% fetal bovine serum (FBS; Gibco-BRL), $100 \mathrm{U} / \mathrm{ml}$ penicillin and $100 \mathrm{mg} / \mathrm{ml}$ streptomycin at $37^{\circ} \mathrm{C}$ in a humidified atmosphere of $5 \% \mathrm{CO}_{2}$.

RNA interference. All siRNAs were designed by GeneChem (Shanghai, China), with sequences as follows: EpCAM siRNA forward, 5'-CGTAAACTGCTTTGTGAATdTdT-3' and reverse, 5'-ATTCACAAAGCAGTTTACGdTdT-3'; and scrambled (SCR) siRNA forward, 5'-ACGUGACACGUUCGGAGA AdTdT-3' and reverse, 5'-UUCUCCGAACGUGUCACG UdTdT-3'.
The FaDu cells were transfected with Lipofectamine 2000 (Invitrogen Life Technologies, Carlsbad, CA, USA) according to the manufacturer's instructions. Briefly, $3.0 \times 10^{4}$ cells were plated in six-well plates (Costar, Cambridge, MA, USA) and cultured at $37^{\circ} \mathrm{C}$ for $\sim 24 \mathrm{~h}$ until $70-80 \%$ confluent. siRNA and Lipofectamine 2000 were then diluted in $300 \mu$ l Opti-MEM reduced serum media (Gibco-BRL). Next, the solutions were mixed together and incubated for $20 \mathrm{~min}$ at room temperature. The cells were then washed twice with Opti-MEM and the mixture solution was transferred to the six-well plates. Following incubation for $8 \mathrm{~h}$, the mixed solution was discarded and $2 \mathrm{ml}$ of $10 \%$ FBS-DMEM was added to each well of the six-well plates. The cells were then incubated at $37^{\circ} \mathrm{C}$ for $\sim 72 \mathrm{~h}$ and lysed with $0.25 \%$ trypsin-EDTA (Gibco-BRL) for subsequent assays.

$R N A$ isolation and quantitative polymerase chain reaction ( $q P C R)$. Total RNA was isolated using TRIzol reagent (Invitrogen Life Technologies). In accordance with the manufacturer's instructions, the first-strand of cDNA was synthesized using the PrimeScript first-strand cDNA synthesis kit [Takara Biotechnology (Dalian) Co., Ltd., Dalian, China] in a reaction mixture with a final volume of $20 \mu \mathrm{l}$, containing $1 \mu \mathrm{g}$ total RNA, $4 \mu 1$ 5X PrimeScript buffer, $1 \mu 1$ deoxynucleotide triphosphate mixture, $1 \mu \mathrm{l}$ Oligo(dT) primer, $0.5 \mu 1$ PrimeScript RTase, $0.5 \mu 1$ RNase inhibitor and RNase-free water. The reverse transcription reaction was performed under the following conditions: $42^{\circ} \mathrm{C}$ for $15 \mathrm{~min}$, followed by a termination step at $95^{\circ} \mathrm{C}$ for $2 \mathrm{~min}$. The qPCR analyses were performed using an Eppendorf Mastercycler (Eppendorf, Westbury, NY, USA). The standard reaction volume was $25 \mu \mathrm{l}$, containing $1 \mu \mathrm{l}$ QuantiTect SYBR Green PCR Master Mix [Takara Biotechnology (Dalian) Co., Ltd], $2 \mu \mathrm{l}$ cDNA template and $0.25 \mu \mathrm{M}$ forward and reverse primers. The initial PCR step was as follows: $2 \mathrm{~min}$ at $50^{\circ} \mathrm{C}$, followed by a $15 \mathrm{~min}$ hold at $95^{\circ} \mathrm{C}$. This was followed by 40 cycles, consisting of a 15 -sec denaturation step at $95^{\circ} \mathrm{C}$, a $20-\mathrm{sec}$ annealing/extension step at $59^{\circ} \mathrm{C}$, and a $72^{\circ} \mathrm{C}$ incubation step for $20 \mathrm{sec}$. All reactions were performed in triplicate. Following normalization to the GAPDH gene, the expression levels for each target gene were analyzed using the comparative threshold cycle (CT) method. The $2^{-\Delta \Delta c t}$ was calculated to determine the relativity using the following formula: $\Delta \Delta c t=\Delta c t($ experimental group $)-\Delta c t$ (control group). The $\Delta$ ct values were calculated using the following formula: $\Delta c t=c t($ target gene $)-\operatorname{ct}($ GAPDH $)$. The EpCAM and GAPDH primers were designed by Takara Biotechnology (Dalian) Co., Ltd., and the primer sequences used were as follows: EpCAM forward, 5'-GAATGG CAAAGTATGAGAAGGCTGA-3' and reverse, 5'-TCCCAC GCACACACATTTGTAA-3'; and GAPDH forward, 5'-CAA GGTCATCCCTGACACTTG-3' and reverse, 5'-GTCCAC CACCCTGTTGCTGTAG-3'.

Protein extraction and western blot analysis. To obtain the total cell lysates, the cells were rinsed twice with ice-cold PBS and lysed in $300 \mu \mathrm{l}$ of hot $\left(100^{\circ} \mathrm{C}\right) 10 \mathrm{mM}$ EDTA containing $1 \%$ sodium dodecyl sulfate (SDS). The detergent-soluble (not anchored to the cytoskeleton) and detergent-insoluble (anchored to the cytoskeleton) proteins were extracted using 
the technique reported by Osta et al (7), with minor revisions. Briefly, the cells were rinsed three times with cold PBS and $300 \mu \mathrm{l}$ cold extraction buffer, which was composed of $50 \mathrm{mM}$ Tris- $\mathrm{HCl}$ (pH 7.0), $50 \mathrm{mM} \mathrm{NaCl}, 3 \mathrm{mM} \mathrm{MgCl}_{2}, 0.5 \%$ Triton X-100, $300 \mathrm{mM}$ sucrose and $1 \%$ protease inhibitor mixture (Sigma-Aldrich, St. Louis, MO, USA). Next, the cells were agitated for $60 \mathrm{~min}$ at $4^{\circ} \mathrm{C}$ and centrifuged in the Sigma 3K15 ultracentrifuge (JinanSigma Zentrifugen $\mathrm{GmbH}$, Osterode, Germany) for $1 \mathrm{~h}$ at $10,000 \mathrm{x} \mathrm{g}$ and $4^{\circ} \mathrm{C}$. The supernatant was collected and the pellet was lysed with $300 \mu \mathrm{l}$ of hot $\left(100^{\circ} \mathrm{C}\right) 1 \% \mathrm{SDS} / 10 \mathrm{mM}$ EDTA and then incubated at $100^{\circ} \mathrm{C}$ for $10 \mathrm{~min}$. The nuclear protein was obtained using the Nuclear Extract kit (Active Motif, Tokyo, Japan) according to the manufacturer's instructions. The protein concentration was then determined by the Enhanced Bicinchoninic Acid Protein Assay kit (Beyotime, Shanghai, China).

The total, soluble, insoluble and nuclear proteins with denaturing conditions were loaded for SDS-polyacrylamide gel electrophoresis (5\% stacking gel and 8\% separating gel), followed by separation at $80 \mathrm{~V}$ for $\sim 30 \mathrm{~min}$ and then $120 \mathrm{~V}$ for $\sim 90 \mathrm{~min}$. The proteins were subsequently transferred to a polyvinylidene difluoride membrane. Following blocking with $5 \%$ skimmed milk/Tris-buffered saline-Tween (TBST) for $1 \mathrm{~h}$ at room temperature, the membranes were incubated with the primary antibodies, rabbit anti-human EpCAM $(1: 2,000)$, rat anti-human E-cadherin (1:2,000), rabbit anti-human $\beta$-catenin $(1: 5,000)$ and mouse anti-human $\beta$-actin $(1: 2,000)$, which were diluted in $3 \%$ skimmed milk/TBST overnight at $4{ }^{\circ} \mathrm{C}$ and then washed three times with TBST for 5 min separately. Subsequently, the membrane was incubated with secondary antibodies, and the signals were visualized by electrochemiluminescence using an LAS-4000 image reader (Fujifilm, Tokoyo, Japan). The EpCAM, E-cadherin, and $\beta$-catenin primary antibodies were purchased from Abcam, whereas the $\beta$-actin primary antibody and all secondary antibodies were purchased from Beijing Zhongshan Golden Bridge Biotechnology Co., Ltd.

In vitro invasion/migration assays. Cell invasion assays were performed using Transwell ${ }^{\mathrm{TM}}$ chambers (Costar, Cambridge, MA, USA). Briefly, subsequent to coating the filter with $50 \mu 1$ Matrigel (BD Biosciences, NY, USA), which had been diluted by DMEM at 1:6 overnight at $37^{\circ} \mathrm{C}$, the upper chamber of the 24-well Transwell plates were filled with $100 \mu \mathrm{l}$ serum-free DMEM containing $1 \times 10^{5}$ cells $/ \mathrm{ml}$. The lower chamber was filled with $0.5 \mathrm{ml}$ DMEM containing $10 \% \mathrm{FBS}$ as a chemical attractant. Following incubation for $24 \mathrm{~h}$ at $37^{\circ} \mathrm{C}$ in a $5 \%$ atmosphere, non-invading cells were removed by scrubbing with a cotton swab. The filters were then fixed with methanol and stained with crystal violet for $15 \mathrm{~min}$. The number of cells that penetrated the filter was quantified under a microscope (magnification, $\mathrm{x} 200$ ). To assess migration, cell migration assays were performed under the same conditions as the Transwell invasion assays without Matrigel-coated Transwell chambers. All experiments were performed in triplicate and repeated three times.

Cell proliferation assay. Briefly, the cells were plated in 96-well plates at a concentration of $5 \times 10^{3}$ cells per well and incubated for 24,48 or $72 \mathrm{~h}$ following the addition of siRNA.
At each time-point, the cells were incubated with $0.5 \mathrm{mg} / \mathrm{ml}$ 3-(4,5-dimethylthiazol-2-yl)-2,5-diphenyltetrazolium bromide (MTT; Sigma-Aldrich). After $4 \mathrm{~h}$, the medium was replaced with $100 \mu \mathrm{l}$ dimethyl sulfoxide (Sigma-Aldrich) and vortexed for $10 \mathrm{~min}$. The absorbance was then recorded at a wavelength of $570 \mathrm{~nm}$ using Thermo Multiskan MK3 (Thermo Fisher Scientific Inc, Waltham, MA, USA).

In vitro colony formation assay. The in vitro colony formation assay was performed to measure oncogenic potential. The control, SCR siRNA and EpCAM siRNA-treated FaDu cells were suspended in 10\% FBS-DMEM, then plated in 6 wells at 500 cells/well with $2 \mathrm{ml}$ DMEM supplemented with $10 \%$ FBS. The number of colonies were counted on the tenth day.

Statistical analysis. Data are presented as the mean \pm standard deviation. Statistical calculations were performed using SPSS version 13.0 (SPSS, Inc., Chicago, IL, USA). The $\chi^{2}$ test, one-way analysis of variance and least significance divergence were applied to analyze the data. $\mathrm{P}<0.05$ was considered to indicate a statistically significant difference, and all tests were two-tailed.

\section{Results}

EpCAM overexpression in primary hypopharyngeal carcinoma. To investigate whether EpCAM is expressed in hypopharyngeal carcinoma, immunohistochemistry was used to analyze the 40 hypopharyngeal carcinoma tissues and 10 normal hypopharyngeal epithelia samples. It was identified that EpCAM was primarily located at the membrane of the hypopharyngeal carcinoma cells, occasionally diffusing into the cytoplasm of cells, however, EpCAM was absent in non-neoplastic tissues (Fig. 1).

EpCAM overexpression correlates with tumor size and lymph node metastasis in hypopharyngeal carcinoma. EpCAM overexpression was markedly increased in the cancer tissues (26 of the 40) when compared with the normal epithelia (0 of the 10) ( $\mathrm{P}=0.001$; Table I). In addition, EpCAM overexpression was found to correlate with tumor size stage $(\mathrm{P}=0.007)$ and lymph node metastasis $(\mathrm{P}=0.029)$.

Downregulation of EpCAM expression significantly decreases the invasion and migration potential of FaDu cells in vitro. Firstly, an EpCAM siRNA was used to silence EpCAM, and the results showed that EpCAM siRNA lead to a marked decrease in EpCAM expression (Fig. 2). To further investigate the effect of EpCAM downregulation on the invasion and migration potential of the FaDu cells, Transwell assays were performed. As indicated in Fig. 3, the number of migratory cells in the EpCAM siRNA treatment groups $(59.83 \pm 8.42)$ decreased significantly when compared with the control $(110.83 \pm 10.01)$ and SCR siRNA treatment $(102.89 \pm 15.55)$ groups $(\mathrm{P}<0.05)$. In the invasion assays, the number of invasive cells in the EpCAM siRNA group $(44.40 \pm 3.75)$ was significantly less than that of the control $(96.61 \pm 10.98)$ and SCR siRNA $(86.64 \pm 5.97)$ groups $(\mathrm{P}<0.05$; Fig. 3). These results revealed that the downregulation of 
Table I. Correlation between EpCAM overexpression and clinicopathological features.

\begin{tabular}{|c|c|c|c|c|}
\hline \multirow[b]{2}{*}{ Characteristics } & \multirow[b]{2}{*}{$\mathrm{n}$} & \multicolumn{2}{|c|}{$\begin{array}{c}\text { EpCAM } \\
\text { overexpression, } \mathrm{n}(\%)\end{array}$} & \multirow[b]{2}{*}{ P-value } \\
\hline & & - & + & \\
\hline Normal epithelium & 10 & $10(100.0)$ & $0(0.0)$ & 0.001 \\
\hline $\begin{array}{l}\text { Hypopharyngeal } \\
\text { carcinoma }\end{array}$ & 40 & $14(35.0)$ & $26(65.0)$ & \\
\hline Gender & & & & 0.652 \\
\hline Male & 38 & $13(34.2)$ & $25(65.8)$ & \\
\hline Female & 2 & $1(50.0)$ & $1(50.0)$ & \\
\hline Age & & & & 0.591 \\
\hline$\geq 60$ & 28 & $10(35.7)$ & $18(64.3)$ & \\
\hline$<60$ & 12 & $4(33.3)$ & $8(66.7)$ & \\
\hline $\mathrm{T}$ classification & & & & 0.007 \\
\hline $\mathrm{T} 1+\mathrm{T} 2$ & 14 & $9(64.3)$ & $5(35.7)$ & \\
\hline $\mathrm{T} 3+\mathrm{T} 4$ & 26 & $5(19.2)$ & $21(80.8)$ & \\
\hline $\mathrm{N}$ classification & & & & 0.029 \\
\hline N0 & 11 & 7 (63.6) & $4(36.4)$ & \\
\hline $\mathrm{N}+$ & 29 & $7(24.1)$ & 22 (75.9) & \\
\hline
\end{tabular}

EpCAM, epithelial cell adhesion molecule.

EpCAM expression inhibits the migration and invasive ability of FaDu cells.

Downregulation of EPCAM expression inhibits the proliferation ability and tumorigenicity of FaDu cells in vitro. To measure the function of EpCAM downregulation on the proliferation ability and tumorigenicity of the FaDu cells in vitro, the MTT and plate colony formation assays were performed. As shown in Fig. 4, in the plate colony assay, the plate colony numbers of the control, SCR siRNA and EpCAM siRNA groups were $177.33 \pm 16.50,173.67 \pm 13.51$ and $78.00 \pm 5.57$, respectively $(\mathrm{P}<0.05)$. In the MTT assay at $48 \mathrm{~h}$ and $72 \mathrm{~h}$, the absorbance of the control $(0.326 \pm 0.017$ for $48 \mathrm{~h}$ and $0.525 \pm 0.017$ for $72 \mathrm{~h})$ and SCR siRNA groups $(0.311 \pm 0.016$ for $48 \mathrm{~h}$ and $0.495 \pm 0.015$ for $72 \mathrm{~h}$ ) were significantly higher than that of the EpCAM siRNA group $(0.256 \pm 0.008$ for $48 \mathrm{~h}$ and $0.335 \pm 0.005$ for $72 \mathrm{~h})(\mathrm{P}<0.05$; Fig. 4C). These results clearly indicated that the downregulation of EpCAM expression inhibits the proliferation ability and tumorigenicity of $\mathrm{FaDu}$ cells.

Downregulation of EpCAM expression increases the E-cadherin, $\alpha$-catenin and $\beta$-catenin expression of the insoluble protein (cytoskeleton). To further investigate the mechanism by which the downregulation of EpCAM expression inhibits the migration and invasion ability, the expression of EpCAM, E-cadherin, $\alpha$-catenin, $\beta$-catenin and $\beta$-actin at the protein level was analyzed by western blot analysis. No evident alterations in E-cadherin, $\alpha$-catenin, and $\beta$-catenin total proteins were observed when compared with the control and SCR siRNA treatment groups (Fig. 5A). However, the EpCAM siRNA treatment gave rise to an apparent increase of E-cadherin, $\alpha$-catenin and $\beta$-catenin in
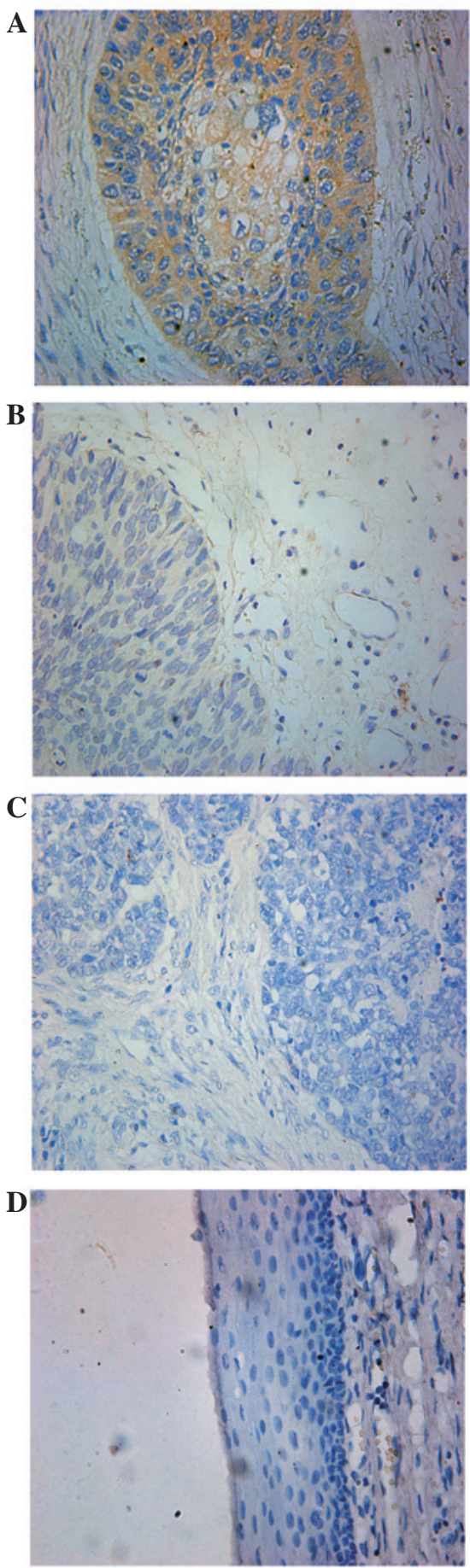

Figure 1. Representative images of EpCAM immunohistochemical staining (magnification, $\mathrm{x} 400$ ). (A) Positive and (B) negative expression of EpCAM in hypopharyngeal carcinoma tissue. (C) Negative control (without EpCAM antibody) pattern and (D) non-cancerous region of hypopharyngeal carcinoma. EpCAM, epithelial cell adhesion molecule.

insoluble protein (cytoskeleton; Fig. 5C) and an apparent decrease of E-cadherin, $\alpha$-catenin and $\beta$-catenin in soluble protein (no anchorage to cytoskeleton; Fig. 5B). These results indicated that EpCAM siRNA treatment possibly enhances the anchorage of E-cadherin, $\alpha$-catenin and $\beta$-catenin to the cytoskeleton.

Downregulation of EpCAM expression inhibits $\beta$-catenin expression in the nucleus. To further investigate the mechanism 
A

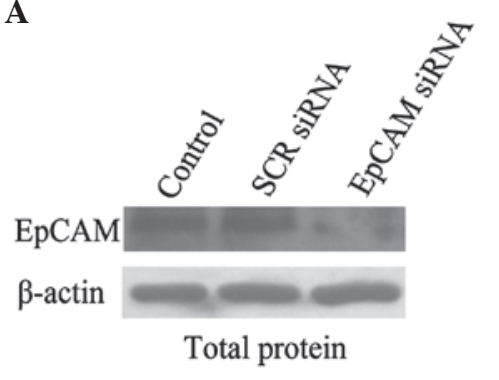

B

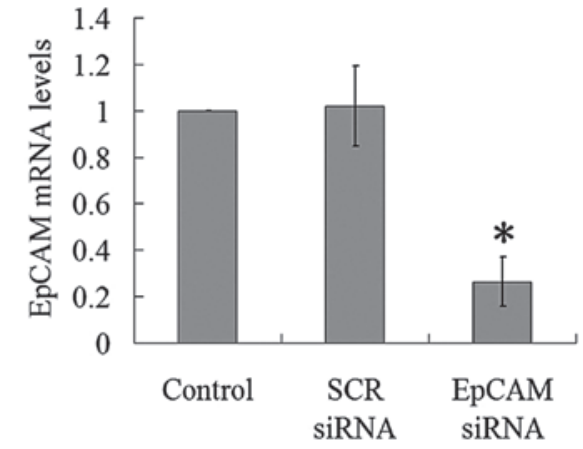

Figure 2. EpCAM siRNA inhibits the expression of EpCAM. Expression of EpCAM in the control, SCR siRNA and EpCAM siRNA groups at the (A) protein and (B) mRNA level. Data are presented as the mean \pm standard deviation of three separate experiments and all experiments were conducted three times. ${ }^{*} \mathrm{P}<0.05$ vs. control/SCR siRNA. EpCAM, epithelial cell adhesion molecule; SCR, scrambled; siRNA, small interfering RNA.

A

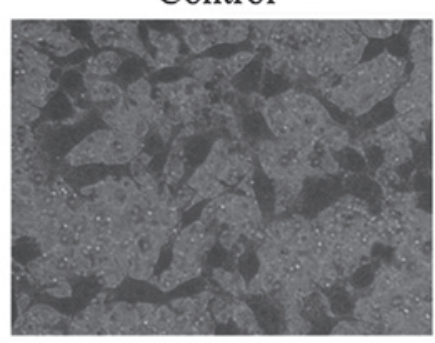

B

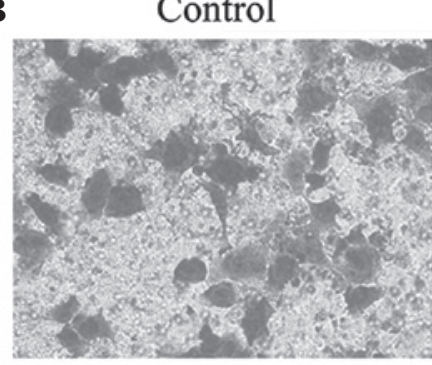

C

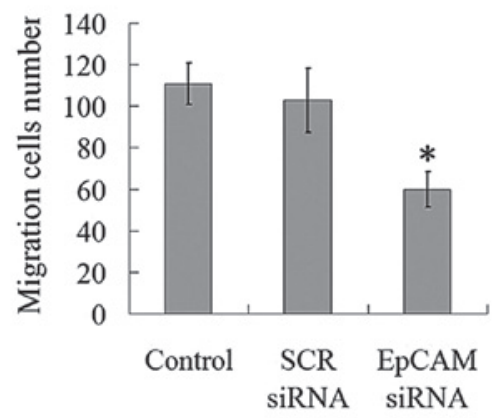

SCR SiRNA

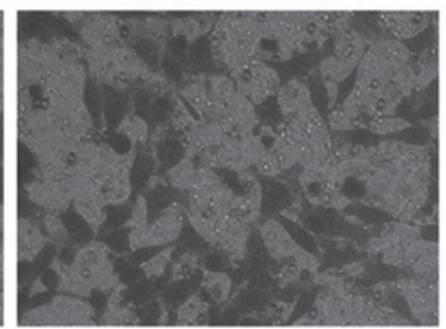

SCR SiRNA

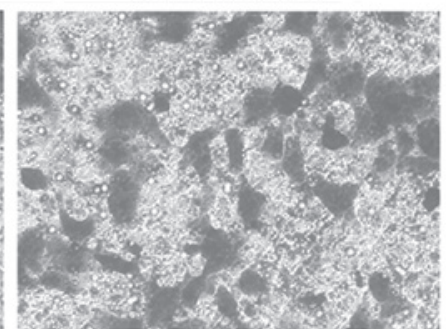

EpCAM siRNA

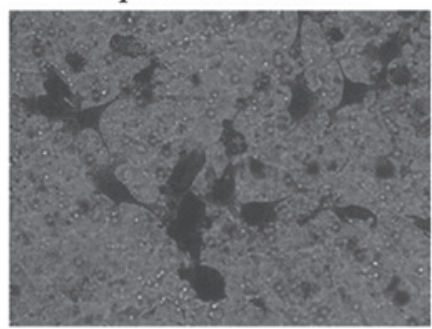

EpCAM siRNA

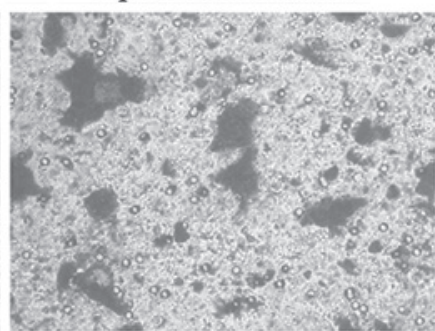

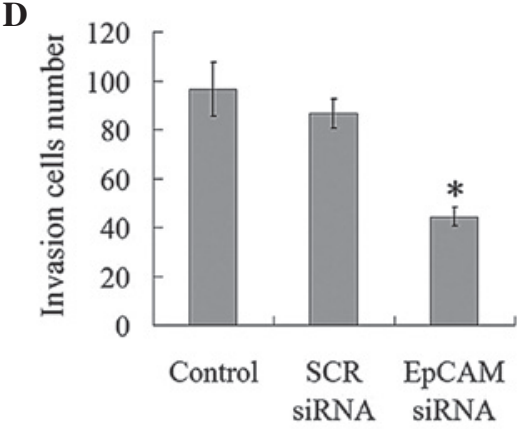

Figure 3. EpCAM siRNA treatment inhibits the migration and invasion of FaDu cells in vitro, as shown by migration and invasion assays. The (A) migration and (B) invasion of the cells were examined by the Transwell chamber assay in the control, SCR siRNA, and EpCAM siRNA groups (magnification, x200). The bar graph presents the numbers of (C) migratory and (D) invasive cells in each group. Data are presented as the mean \pm standard deviation of three separate experiments and all experiments were conducted three times. ${ }^{~} \mathrm{P}<0.05$ vs. control/SCR siRNA. EpCAM, epithelial cell adhesion molecule; siRNA, small interfering RNA; SCR, scrambled.

by which the downregulation of EpCAM expression inhibits the proliferation and tumorigenicity, the expression of $\beta$-catenin in the nucleus was analyzed. The results revealed that the downregulation of EpCAM decreases the expression of $\beta$-catenin in the nucleus (Fig. 5D).

\section{Discussion}

Although EpCAM has been well studied as a cancer-associated antigen, the clinicopathological significance of EpCAM overexpression in cancer remains unclear. On the one hand, certain 
A
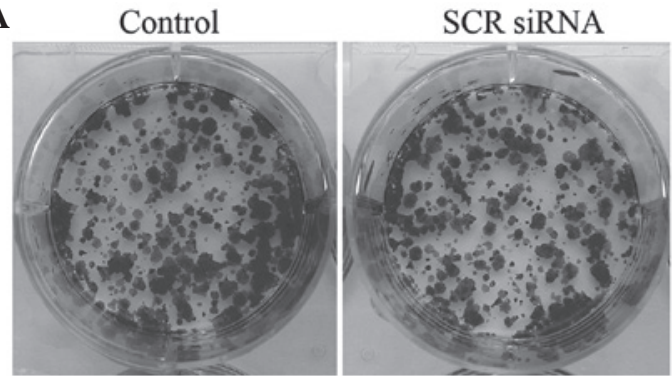

EpCAM siRNA

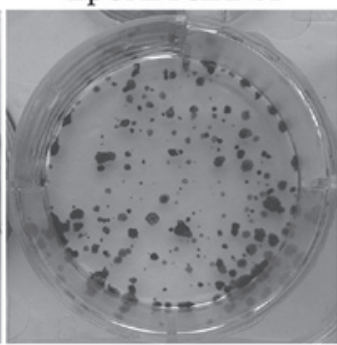

B

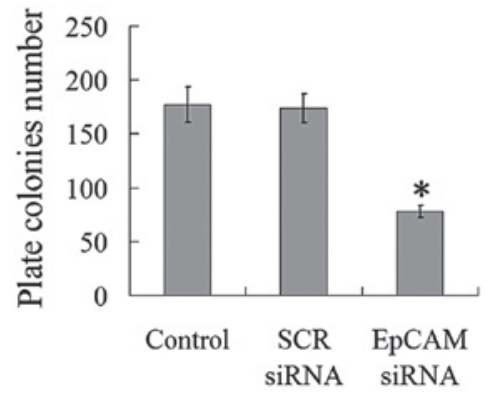

C

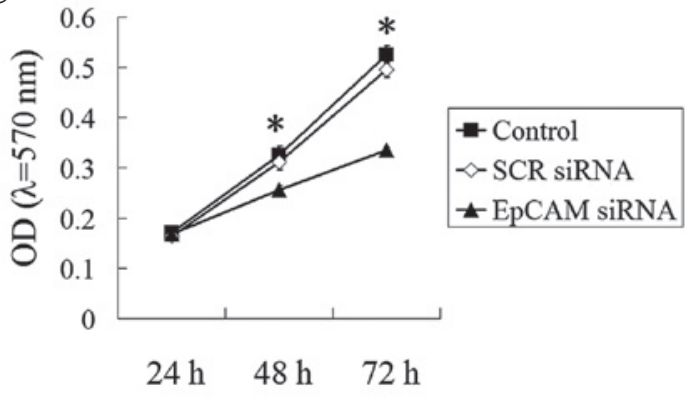

Figure 4. EpCAM siRNA treatment inhibits the proliferation ability and tumorigenicity of FaDu cells in vitro. (A) Representative images of the plate colony assay in the control, SCR siRNA and EpCAM siRNA groups. (B) Bar graph presenting the plate colony numbers of each group. (C) Growth curves for each group by MTT assay. Data are presented as the mean \pm standard deviation of three separate experiments and all experiments were conducted three times. ${ }^{2} \mathrm{P}<0.05$ vs. control/SCR siRNA. EpCAM, epithelial cell adhesion molecule; SCR, scrambled; siRNA, small interfering RNA; OD, optical density.

A

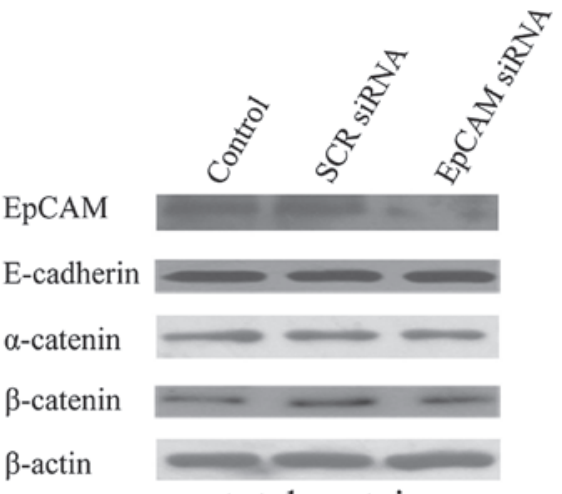

C

EpCAM

E-cadherin

$\alpha$-catenin

$\beta$-catenin

$\beta$-actin total protein

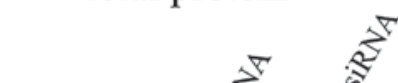

B

EpCAM

E-cadherin

$\alpha$-catenin

$\beta$-catenin

$\beta$-actin

\section{soluble protein}

D

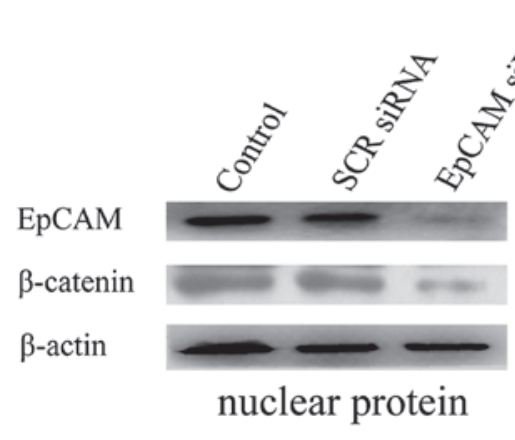

Figure 5. EpCAM siRNA treatment regulates the expression of E-cadherin, $\alpha$-catenin and $\beta$-catenin. (A-C) Alterations in the expression of E-cadherin, $\alpha$-catenin and $\beta$-catenin at total, soluble and insoluble protein levels in the control, SCR siRNA and EpCAM siRNA groups. (D) Alteration of $\beta$-catenin at the nuclear level in each group. EpCAM, epithelial cell adhesion molecule; SCR, scrambled; siRNA, small interfering RNA.

studies have reported that the expression of EpCAM improves the patient survival rate in specific cancer types, including renal cell and thyroid cancers (18). On the other hand, in gastric, breast and tongue cancer, high EpCAM expression has been identified as an indicator of advanced stage and poor prognosis $(7,19,20)$. Various biological explanations may be 
indicated for these mixed results, including the possibility that EpCAM could exhibit varying functions in different organs and affected tissues.

The present study investigated the expression pattern of EpCAM in hypopharyngeal carcinoma. EpCAM overexpression was observed in the majority of the hypopharyngeal carcinoma tissues, whereas it was absent in the normal hypopharyngeal epithelia. Furthermore, a significant correlation was identified between high EpCAM expression and advanced tumor size stage or lymph node metastasis. To further confirm the results obtained by immunohistochemical staining, a series of in vitro assays were performed in the hypopharyngeal carcinoma FaDu cell line. The silencing of EpCAM expression using siRNA was found to suppress the invasion, migration, proliferation and tumorigenicity of the FaDu cells. Therefore, the aforementioned observations in the tissues and cell lines provides compelling evidence that EpCAM expression promotes hypopharyngeal carcinoma progression and metastasis.

The mechanism by which EpCAM enhances metastasis in cancer remains unclear. EpCAM is a $\mathrm{Ca}^{2+}$-independent homophilic CAM that early studies in cells deficient in intercellular adhesion interactions, such as mouse fibroblast cell lines, have found to be able to mediate cell aggregation, prevent cell scattering and also direct cell segregation when introduced into cells (21). Based on these adhesive functions, EpCAM was considered to be an inhibitor of tumor metastasis, which appears to present a paradox. Further understanding of EpCAM regulation on E-cadherin mediated-adhesion has clarified this subject. In the epithelium, E-cadherin molecules function by connecting neighboring cells, thus forming a bridge between their cortical actin cytoskeleton to maintain mechanical coupling within the cells and establish intercellular adhesion (22). Furthermore, the regulation of the intercellular adhesion is dependent on the recruitment of $\alpha$-catenin and $\beta$-catenin to the adhesion sites of the adhesion complexes, and their anchorage to the actin cytoskeleton. Winter et al (14) revealed that EpCAM expression inhibits E-cadherin-mediated cell-to-cell adhesion by disrupting the link between $\alpha$-catenin and F-actin. In this manner, EpCAM relaxes the tight intercellular adhesions and promotes metastasis, differentiation and tissue maintenance. In addition, Osta et al (7) reported a similar phenomenon in breast cancer. In the present study, it was observed that the downregulation of EpCAM improves the anchorage of E-cadherin, $\alpha$-catenin and $\beta$-catenin to the actin cytoskeleton (insoluble protein fraction) in FaDu cells, while the expression of these proteins in the cytoplasm (soluble protein fraction) was markedly decreased. Thus, we hypothesize that in hypopharyngeal carcinoma, the downregulation of EpCAM expression inhibiting tumor invasion and migration may transfer cytoplasmic E-cadherin, $\alpha$-catenin and $\beta$-catenin anchorage to the actin cytoskeleton, tightening cell-to-cell adhesion.

However, the mechanism by which EpCAM expression contributes to proliferation and colony formation is not entirely understood. Chaves-Pérez et al (23) recently provided evidence that EpCAM overexpression induces the rapid upregulation of the oncogenes c-myc and cyclin D1, which induce cellular proliferation. These transcriptional factors are activated by the nuclear translocation of $\beta$-catenin. De novo expression of EpCAM in HKE293 cells induces a redistribution of $\beta$-catenin from the cytoplasm to the nucleus (15). Maetzel et al (15) further investigated the association between EpCAM and $\beta$-catenin, and the cleavage of EpCAM led to EpCAM EpICD nuclear translocation in a complex with $\beta$-catenin and T-cell factor. Within the nucleus, the EpICD complex interacts with Lef-1 and contacts DNA to activate the target genes. In the present study, the downregulation of EpCAM expression was found to result in the decreased expression of $\beta$-catenin in the nucleus of the FaDu cells, which is consistent with the findings in the HKE293 cells. This may confirm that the downregulation of EpCAM leads to a decrease of the complex and $\beta$-catenin in the nucleus, and the subsequent inactivation of c-myc, cyclin D1 and other target genes, in order to inhibit the cell proliferation and colony formation ability. Therefore, the aforementioned results may account for the mechanism of EpCAM expression promoting $\mathrm{FaDu}$ cell proliferation and colony formation.

In conclusion, the results of the present study demonstrated for the first time that EpCAM is overexpressed in hypopharyngeal carcinoma and that EpCAM expression is tightly associated with tumor size and lymph node metastasis. Furthermore, silenced EpCAM may suppress the invasion, migration, proliferation and colony abilities of hypopharyngeal carcinoma in vitro. This study clearly indicates that EpCAM is a promising target for hypopharyngeal carcinoma therapy.

\section{Acknowledgements}

The present study was supported by the Shandong Provincial International Science and Technology Cooperation Project of China (grant no. 2010GHZ20202).

\section{References}

1. Clark JI, Hofmeister C, Choudhury A, et al: Phase II evaluation of paclitaxel in combination with carboplatin in advanced head and neck carcinoma. Cancer 92: 2334-2340, 2001.

2. Hoffman HT, Porter K, Karnell LH, et al: Laryngeal cancer in the United States: changes in demographics, patterns of care, and survival. Laryngoscope 116 (Suppl 111): S1-S13, 2006.

3. Ma J, Lu S, Yu L, et al: FaDu cell characteristics induced by multidrug resistance. Oncol Rep 26: 1189-1195, 2011.

4. Baeuerle PA and Gires O: EpCAM (CD326) finding its role in cancer. Br J Cancer 96: 417-423, 2007.

5. Schnell U, Cirulli V and Giepmans BN: EpCAM: structure and function in health and disease. Biochim Biophys Acta 1828: 1989-2001, 2013

6. Went P, Vasei M, Bubendorf L, et al: Frequent high-level expression of the immunotherapeutic target Ep-CAM in colon, stomach, prostate and lung cancers. Br J Cancer 94: 128-135, 2006.

7. Osta WA, Chen Y,Mikhitarian K, et al: EpCAM is overexpressed in breast cancer and is a potential target for breast cancer gene therapy. Cancer Res 64: 5818-5824, 2004.

8. Flatmark K, Borgen E, Nesland JM, et al: Disseminated tumour cells as a prognostic biomarker in colorectal cancer. Br J Cancer 104: 1434-1439, 2011.

9. Ni J, Cozzi PJ, Duan W, et al: Role of the EpCAM (CD326) in prostate cancer metastasis and progression. Cancer Metastasis Rev 31: 779-791, 2012.

10. Ji J, Yamashita T, Budhu A, et al: Identification of microRNA-181 by genome-wide screening as a critical player in EpCAM-positive hepatic cancer stem cells. Hepatology 50: 472-480, 2009.

11. Pauli C, Münz M, Kieu C, et al: Tumor-specific glycosylation of the carcinoma-associated epithelial cell adhesion molecule EpCAM in head and neck carcinomas. Cancer Lett 193: 25-32, 2003.

12. Litvinov SV, Velders MP, Bakker HA, Fleuren GJ and Warnaar SO: Ep-CAM: a human epithelial antigen is a homophilic cell-cell adhesion molecule. J Cell Biol 125: 437-446, 1994. 
13. Balzar M, Prins FA, Bakker HA, Fleuren GJ, Warnaar SO and Litvinov SV: The structural analysis of adhesions mediated by Ep-CAM. Exp Cell Res 246: 108-121, 1999.

14. Winter MJ, Nagelkerken B, Mertens AE, Rees-Bakker HA, Briaire-de Bruijn IH and Litvinov SV: Expression of Ep-CAM shifts the state of cadherin-mediated adhesions from strong to weak. Exp Cell Res 285: 50-58, 2003.

15. Maetzel D, Denzel S, Mack B, et al: Nuclear signalling by tumour-associated antigen EpCAM. Nat Cell Biol 11: 162-171, 2009.

16. Visvader JE and Lindeman GJ: Cancer stem cells in solid tumours: accumulating evidence and unresolved questions. Nat Rev Cancer 8: 755-768, 2008.

17. Gires O, Klein CA and Baeuerle PA: On the abundance of EpCAM on cancer stem cells. Nat Rev Cancer 9: 143, 2009.

18. van der Gun BT, Melchers LJ, Ruiters MH, de Leij LF, McLaughlin PM and Rots MG: EpCAM in carcinogenesis: the good, the bad or the ugly. Carcinogenesis 31: 1913-1921, 2010.
19. Kroepil F, Dulian A, Vallböhmer D, et al: High EpCAM expression is linked to proliferation and lauren classification in gastric cancer. BMC Res Notes 6: 253, 2013.

20. Yanamoto S, Kawasaki G, Yoshitomi I, Iwamoto T, Hirata K and Mizuno A: Clinicopathologic significance of EpCAM expression in squamous cell carcinoma of the tongue and its possibility as a potential target for tongue cancer gene therapy. Oral Oncol 43: 869-877, 2007.

21. Litvinov SV, Bakker HA, Gourevitch MM, Velders MP and Warnaar SO: Evidence for a role of the epithelial glycoprotein 40 (Ep-CAM) in epithelial cell-cell adhesion. Cell Adhes Commun 2: 417-428, 1994.

22. Dufour S, Mège RM and Thiery JP: $\alpha$-catenin, vinculin, and F-actin in strengthening E-cadherin cell-cell adhesions and mechanosensing. Cell Adh Migr 7: 345-350, 2013.

23. Chaves-Pérez A, Mack B, Maetzel D, et al: EpCAM regulates cell cycle progression via control of cyclin D1 expression. Oncogene 32: 641-650, 2013. 